\title{
Diffuse esophageal parakeratosis
}

A 31-year-old woman, who suffered from chronic alcohol abuse, presented with hematemesis and loss of consciousness 2 hours after ingesting toilet bowl cleaner and sedative pills. Over the previous 6 months she had suffered worsening depressive symptoms, with increased alcohol intake, inadequate diet, and a rapid weight loss of $20 \mathrm{~kg}$. An urgent endoscopy revealed extensive gastric and duodenal hemorrhage as a result of caustic injury. The esophagus was not a source of bleeding; however, it showed an unusual appearance of thick mucosa with orderly displayed rings and furrows (Figure $\mathbf{1} \mathbf{a}$ ). The furrows were particularly prominent when the lumen constricted upon irrigation (Figure $\mathbf{1 b}$ ). The lesion started with discrete patches at the upper esophagus (Figure 1c), extended diffusely through the entire esophagus, and ended up at the esophagogastric junction. A section of square-shaped mucosa at the upper esophagus was peeled off by random grasping using biopsy forceps (Figure $\mathbf{1} \mathbf{d}$ ). Epithelial parakeratosis was demonstrated by microscopic examination (Figure 2 ). The patient was treated with alcohol abstinence, proton-pump inhibitor, standard tube-feeding diet, and supplements of thiamine, pyridoxine, and cyanocobalamine. A follow-up endoscopy 40 days later revealed normal esophageal mucosa except for scattered candidal infections.

Diffuse esophageal hyper-/parakeratosis is an uncommon endoscopic finding. It is associated with conditions like tylosis, mucosal hyperkeratosis syndrome, and pachyonychia congenita [1]. In addition to genetic diseases, it may be linked to ethanol exposure, duodenal reflux, riboflavin deficiency, and zinc deficiency [25]. Rapid and complete recovery after nutritional supplement in this patient suggests a causal-result relationship between them.

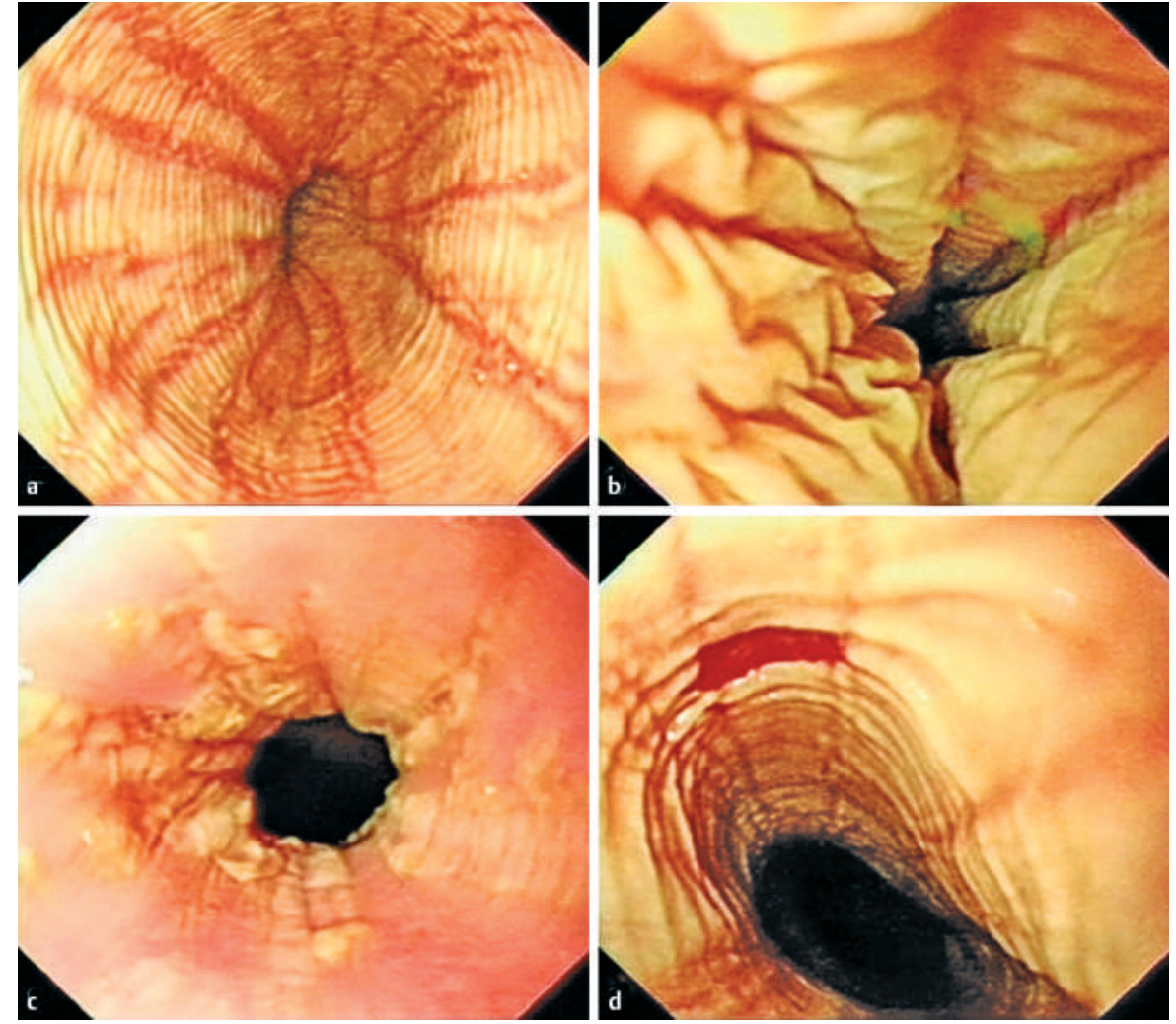

Figure 1 Endoscopic finding of diffuse esophageal mucosal thickening. a Thick mucosa with orderly displayed rings and furrows. b Prominent furrows during lumen constriction. c The upper esophagus showing discrete lesion patches. d Random grasping with biopsy forceps removed a square-shaped section of mucosa at the upper esophagus.

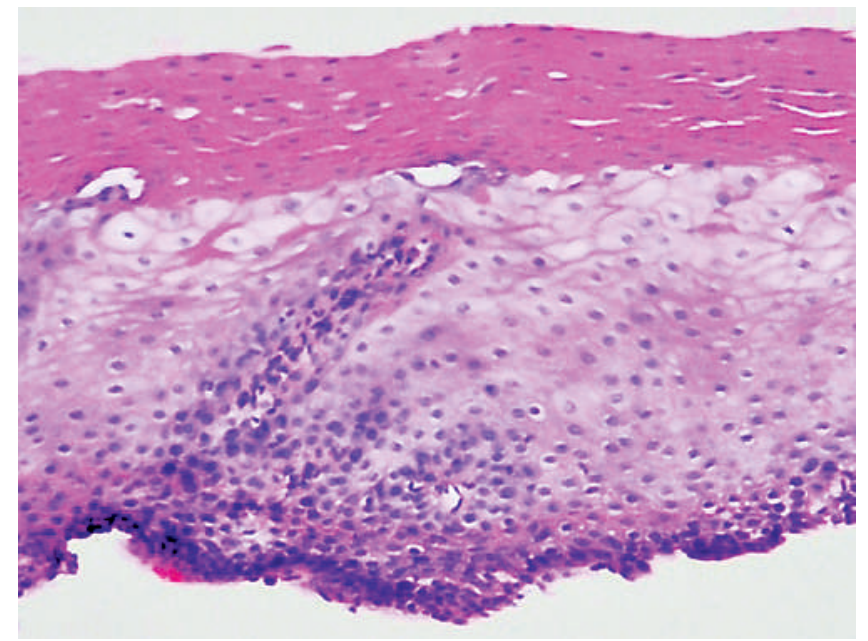

Figure 2 Biopsy of upper esophagus reveals a dense layer of parakeratosis covering the normal-appearing squamous epithelium (hematoxylin and eosin, original magnification $\times 100$ ). 


\section{UCTN}

Endoscopy_UCTN_Code_CCL_1AB_2AC_3AH

\section{H. Tu' ${ }^{1}$ C. M. Tai' ${ }^{1}$ C. Y. Chang1,}

T. H. Chiang', C. T. Lee', J. T. Lin ${ }^{2}$

${ }^{1}$ Department of Internal Medicine, E-DA Hospital, Kaohsiung, Taiwan

2 Department of Internal Medicine, National Taiwan University Hospital and National Taiwan University College of Medicine, Taipei, Taiwan.
${ }^{1}$ Ashworth MT, Nash JR, Ellis A et al. Abnormalities of differentiation and maturation in the oesophageal squamous epithelium of patients with tylosis: morphological features. Histopathology 1991; 19: 303-310

${ }^{2}$ Korsten MA, Worner TM, Feinman L et al. Balloon cytology in screening of asymptomatic alcoholics for esophageal cancer, Part I. Dig Dis Sci 1985; 30: 845-851

${ }^{3}$ Clark GW, Smyrk TC, Mirvish SS et al. Effect of gastroduodenal juice and dietary fat on the development of Barrett's esophagus and esophageal neoplasia: an experimental rat model. Ann Surg Oncol 1994; 1: 252 -261

${ }^{4}$ Foy $\mathrm{H}$, Kondi $\mathrm{A}$. The vulnerable esophagus: riboflavin deficiency and squamous cell dysplasia of the skin and the esophagus. J Natl Cancer Inst 1984; 72: 941 -948

${ }^{5}$ Barney GH, Orgebin-Crist MC, Macapinalac MP. Genesis of esophageal parakeratosis and histologic changes in the testes of the zinc-deficient rat and their reversal by zinc repletion. J Nutr 1968; 95: 526-534
Corresponding author

\section{J. T. Lin, MD}

Department of Internal Medicine National Taiwan University Hospital 7 Chung-Shan South Road

Taipei

Taiwan 10016

Fax: $\quad+886-2-23947899$

Email: jawtown@ha.mc.ntu.edu.tw 